\title{
What standards and terms of employment should respiratory practitioners with a special interest expect from an employing organisation?
}

\section{*Kevin Gruffydd-Jones ${ }^{a}$, Paul Stephenson ${ }^{b}$, Mark L Levyc on behalf of the GPIAG Working Party}

a GPIAG, Hon Lecturer Universities of Aberdeen and Bath, The Surgery, London Road, Box, Wiltshire, UK

${ }^{b}$ Deputy Editor, PCRJ, The Christmas Maltings and Clements Practice, Haverhill, Suffolk, UK

Editor-in-Chief, PCRJ and GPIAG, Senior Clinical Research Fellow, Allergy and Respiratory Research Group, Division of Community Health Sciences:GP Section, University of Edinburgh, UK

Received 30th November 2006; accepted 4th March 2007

\begin{abstract}
Summary model.

(C) 2007 General Practice Airways Group. All rights reserved.

K Gruffydd-Jones, et al. Prim Care Resp J 2007; 16(3): 182-187.

doi:10.3132/pcrj.2007.00032
\end{abstract}

The UK Department of Health (DoH), with input from with the Royal College of General Practitioners and UK General Practice Airways Group (GPIAG), has drawn up guidelines for the appointment of General Practitioners or other Practitioners with a Special Interest (GPwSIs or PwSIs, respectively) in respiratory medicine. This Discussion paper is based on the deliberations of a GPIAG-organised multiprofessional Working Party held in November 2005. It highlights the three key roles of respiratory GPwSIs/PwSIs, and it aims to provide existing and potential specialist practitioners with a checklist of specific points to consider when drawing up a contract with their employing authority. Whilst this system is particular to the provision of primary care in the UK, other countries may choose to follow this

Keywords respiratory, practitioners, general practitioners, special interest, terms of employment

\section{Introduction}

The UK Department of Health (DOH), together with the Royal College of General Practitioners (RCGP) and the UK General Practice Airways Group (GPIAG), has published guidelines for the appointment of practitioners with a special interest in respiratory medicine, and these specialist practitioners may be either general practitioners (GPwSIs) or other allied healthcare practitioners (PwSIs). ${ }^{1}$ Williams et al were early proponents of respiratory GPwSIs, and in their paper published five years ago they explored a number of models for the implementation of the GPwSI role in the care of respiratory patients. ${ }^{2}$ Subsequently, the appointment and role of GPwSls or PwSIs now varies across the UK. The RCGP and DOH are currently drawing up generic guidelines for the appointment of GPwSls, incorporating many of the recommendations mentioned in the
DOH/RCGP/GPIAG document.' It is now well recognised that the individual GPwSI needs to be 'fit for post' (i.e. accredited ${ }^{3}$ ) but also that the post itself needs to be fit for a GPwSI.

In this Discussion paper, we present the findings of a multi-professional Working Party organised by the GPIAG in November 2005 which focussed on the terms of service which a respiratory GPwSI or PwSI should be offered before taking up employment. Much of this paper therefore serves as a checklist for existing or potential practitioners when entering into a contract of employment with an employer. Nowadays, in the UK, the employer might be a Primary Care Trust (PCT), an alternative primary care organisation (PCO), a Commissioning Group, or a private provider.

For ease of reference we will use the generic term 'PwSI' to refer to all types of practitioners - including GPs, specialist

*Corresponding author: Tel: +44 (0)1225 742361 Fax: +44 (0)1225 742646 Email: Kevin.Gruffydd-Jones@gp-j83013.nhs.uk 
nurse practitioners and other healthcare professionals - all of whom can have a specific role as a 'special interest' practitioner in respiratory medicine.

\section{The role of respiratory PwSls}

The DOH document ${ }^{1}$ outlined the three key roles of a PwSI as:

- Service Development and Leadership

- Provision of Specialist Clinical Services

- Education and Liaison

In the absence of specific guidance for respiratory PwSIs the GPIAG has developed and published a series of materials to support respiratory PwSIs. This includes guidance on accreditation, ${ }^{3}$ appraisal, ${ }^{4}$ and also a resource pack which was sent to all PCOs in the United Kingdom. ${ }^{5}$

The employing authority may wish to incorporate some or all of these three key roles into the job description for the PwSI. The job description, and therefore the terms of service, will almost certainly be tailored according to the needs of the employing authority.

\section{Three service framework models for PwSIs}

Before appointing a PwSI, the employing authority may have undertaken a full and comprehensive healthcare needs assessment, reviewed resource implications, developed a service framework model, incorporated a quality assurance system, and addressed governance issues. However, this might be one of the roles required of the PWSI - in which case this should be included in the contract.

In the majority of cases, the specific PwSI service model to be adopted by the PCO should be informed by the outcomes of a healthcare needs assessment performed within the specific health economy. By undergoing this analysis, deficiencies across the care pathway will have been identified and an appropriate service then designed. Within the UK National Health Service (NHS) there are in reality three different PwSI service models in operation. The 'operational/clinical service' model focuses mostly on specific patient groups who have been identified as receiving suboptimal care. This model may incorporate a triage and clinic approach, or specific clinics targeted at "at risk" groups. The 'strategic model' identifies the PWSI as a 'clinical champion' involved in a leadership capacity in redesigning clinical pathways thereby ensuring better care for patients. However, many PwSI services will incorporate both of these roles within a third 'hybrid' model: this hybrid model conforms most clearly to the three key roles identified by the DOH previously. However, it is important for organisations to understand that one size does not fit all and that the relative mix of the three roles must reflect the needs of the specific health community within which it is established.

Once the PwSI model is determined, issues need to be addressed concerning quality assurance of the service. A robust governance structure needs to be incorporated in the model, thereby ensuring accountability and minimal risk to the employing organisation.

Resources required to ensure the success of the service need to be acknowledged and commissioned as part of the service, e.g. accommodation, provision of information technology, support staff, training and mentoring of staff.

The PwSI service should be performance-managed against set objectives determined following the health needs assessment. Thus, performance indicators designed to demonstrate better patient care in a cost effective environment will ensure sustainability of the service. All these issues must be addressed within the business proposal to be presented to the commissioning organisation.

We believe that the most productive outcomes will result when the appointed PwSI is part of a multi-professional team (MPT) who share responsibility for managing difficult, complex, chronic conditions which exceed the ability and resources of the everyday practising GP. The MPT should work across the primary and secondary care interface and should have a clear precise remit, caseload, and reporting structure.

The role of the respiratory PWSI will therefore be a continually evolving position which must respond to the needs of the wider community in which it serves. In many cases the role will develop over time - from a largely clinical role into one which has a greater input in terms of education and service development.

\section{Scope of the service to be provided by the PwSI}

The scope of the service to be provided by the PwSI will depend to a large degree on the employing authority and will be driven by local demand and the abilities and skills of the person appointed. In some cases there may only be a need for a practitioner with specific skills in speciality subsets, e.g. paediatric asthma, but this must be clearly defined. In many cases, however, the employing authority will be looking for a team who will manage long-term complex conditions such as asthma and COPD and provide leadership and education to many different groups within the community. The key aspects of the scope of the service to be provided by a respiratory PwSI correspond to the $\mathrm{DOH}$ document $^{1}$ and fall into the following categories: clinical services; leadership and service development; education; and research and evaluation. The checklists regarding these different categories are shown in Box 1.

\section{Accreditation and appraisal for PwSls}

The GPIAG have suggested that accreditation of the PwSI should occur via portfolio. ${ }^{3}$ Compulsory accreditation is being considered by the DOH with effect from the end of 2007 
Box 1. Checklist regarding the scope of the PwSI service.

\section{a) Clinical services:}

This section deals with the clinical caseload (likely to be COPD and asthma, but the caseload may include sleep apnoea assessment, for example), the setting in which the PwSI will work, the diagnostic and treatment facilities available, the expectation of any specialist skills which the PwSI may be expected to perform, and the role of the PwSI within the clinical team and care pathway.

What conditions are expected to be dealt with?

e.g. COPD, Asthma, TB, all respiratory conditions

Who are the patient types to be seen?

e.g. paediatrics, adults, elderly, all age groups?

In what setting are the patients to be seen?

e.g. traditional primary care, secondary care clinic, cottage hospital

Are the number of clinical sessions sufficient to maintain clinical competence?

e.g. normally at least 1 session/week

Are the length of appointments appropriate to allow delivery of the job plan?

What is the anticipated caseload?

e.g. new patients/follow-ups, "one-off diagnose and treat", or ongoing

What are the approved referral protocols (from primary care and to secondary care)?

Can the PwSI refer direct to secondary care without going back to the patient's GP?

What access is there to investigations and tests?

The minimum expectation should be availability for spirometry, pulse oximetry and allergy testing (if providing an allergy service).

Is there access to diagnostic services and reporting (X-ray and ECG)?

Is there a fast-track process available for diagnostic services?

What are the facilities to be provided?

Where will they be provided?

Are they appropriate to allow delivery of the expectations of the job plan?

Are the facilities safe, clean and compliant to health and safety regulations?

What is the criteria for admission, and availability of, intermediate care and secondary care?

Is there direct access to the secondary care respiratory team and an immediate/urgent second opinion?

What support staff are available to support the PwSI in this role according to need? e.g. administrative support, nursing support, physiotherapy, etc.

What are the arrangements for cover during unplanned absence, sickness etc?

What are the processes involved for appropriate record keeping? Do record-keeping systems link with hospital, Out of Hours services $(\mathrm{OOH})$, General Practices, Nursing staff? Are record-keeping services in line with good medical practice?

Are there arrangements for prescribing?

What are the prescribing restrictions?

Is there a local prescribing formulary/guidelines? Whose budget does the PwSI's salary come out of?

What are the responsibilities for other prescribers within the respiratory team?

To whom is the PwSI accountable in this element of the role?

Box 1 continued overleaf 
Box 1. Checklist regarding the scope of the PwSI service continued.

\section{b) Leadership and service development:}

This section deals with the PwSI's role in developing community respiratory services, and the clarification of their role within the respiratory team.

What is the PwSI's role as a clinical lead?

Do I have an advisory role and if so to whom? e.g. PEC/other committees, patient groups, primary care, secondary care.

Do I have a liaison role and if so with whom? e.g. coordination of care, PEC/other committees, primary care, secondary

care, patient groups etc.

Do I have a strategic role?

i. Understanding local and national service frameworks,

ii. Implementing local/national service framework,

iii. ? public health role

Do I have a commissioning role and if so what is the role (e.g. leader, advisor, part of a team)?

Do I have access to the PCO service development/commissioning team in this part of my role?

What support is available to me in the overall service development/leadership role? e.g. PEC chair, Chief Executive, Secondary care colleagues, administrative support, human resources

i) Will I have access to meeting rooms, facilities and equipment (e.g. computers) to fulfil this element of my role? Whom am I accountable to in this part of my role? e.g. Medical Director, Chief Executive.

ii) How much time am I expected to spend on this element of my role?

\section{c) Education}

The PwSI should establish whether or not they will be involved in planning and effecting health strategy to other healthcare professionals, patient groups, or non-clinicians.

Is there an education strategy?

Will I be required to advise on education requirements/strategy?

Will I be expected to be involved in the provision of education and if so to whom and in what format (groups, one-to-one)?

Will I be expected to take a governance role in terms of education?

What support will there be to assist me in this role? e.g. administration, facilities etc.

What are the expectations in terms of time commitment from the employing authority?

\section{d) Research and evaluation}

The PwSI may need to be able to evaluate the services provided. The scope of such an evaluation, together with the time required to perform it, will need to be stipulated.

Are systems in place to encourage/promote the development of respiratory research programmes?

What research opportunities will be available to help me to evaluate the services provided?

(see below). The key elements of accreditation by portfolio are:

- the track record of the PwSI

- evidence of a personal development plan in respiratory medicine

- proven analytical reflection on clinical work

- submission of a proposal for improvement of respiratory care within a PCO/evidence of knowledge of the principles involved. An example of the minimum level of knowledge required for a GPwSI in allergy has been described previously. ${ }^{6}$
- evidence of educational skills

- evidence of clinical skills

A diploma in primary care respiratory medicine (for example, as provided by Education for Health ${ }^{7}$ ), while not essential, could be used as evidence of acquisition of many or all of these key elements.

In addition, as part of good governance practice there is a clear need for PwSls to have a formal system of appraisal which forms part of the professional development process. The GPIAG has proposed a model for appraisal of GPwSls in respiratory medicine. ${ }^{4}$ 
Box 2. Clinical governance issues.

Is there a structure for managing critical events?

What is the process for complaints?

What are the disciplinary procedures and policies?

Is there a job plan?

How often is the job plan reviewed/amended?
To whom is the PwSI accountable for both clinical and managerial roles?

How will the success of the role be evaluated?

How will performance be measured/judged?

Are there clearly defined performance indicators?

Who audits the service?
Therefore, for the aspiring PwSI, the questions relating to accreditation are as follows: what is the accreditation process and does it conform to recognised guidelines? (for example, GPIAG guidance, ${ }^{3}$ or the UK General Medical Council); who undertakes the accreditation process (specialist clinicians, other GPwSls, or a local GP?) and how often does it take place?; are there any confidentiality issues with regard to the employing organisation?; is there a period of 'apprenticeship' permitted which incorporates protected study-time?; can the PwSI be employed whilst training?; and, if so, is this paid by the employing authority?

In terms of ongoing appraisal, the questions to be considered are: is there a system in place for regular appraisal and, if so, how often is it undertaken?; who undertakes the appraisal process and are they trained in appraisal skills?; does the appraisal system meet approved standards e.g. GPIAG recommendations for the appraisal of respiratory GPwSIs?:4 is time allowed for preparation and the appraisal?; and what actions can be agreed following appraisals?

\section{Professional support}

Ongoing professional support is of particular importance to respiratory PwSIs, some of whom may feel quite isolated at times. It is important to find out who is going to be providing the ongoing professional support, and what the arrangements are for specialist training - for example, parallel clinics, multidisciplinary team meetings, mentorship etc. It is also essential to ascertain whether or not there are arrangements for continuing professional development (CPD) and whether or not there is protected time and funding allowed for CPD.

\section{Clinical governance}

Increasingly, governance issues are paramount in the development and implementation of a new role. In addition to encompassing legislative procedures and ensuring the best interests of the patient, employee and employer governance should also incorporate appropriate measures for evaluation of the role. Questions relating to clinical governance issues which need to be considered are shown in Box 2 .

\section{Multi-professional working}

The GPIAG Working Party believes that the best outcomes for patients and employers will occur as a result of the PwSI working as part of a multi-professional team (MPT) working across the primary and secondary care interface. The following questions outline areas for consideration in ensuring appropriate team support:

- Is there a clearly defined MPT?

- Are the roles within the team defined?

- What is the role of the PWSI within the respiratory team(s)?

- What level of support/involvement is there from secondary care respiratory colleagues?

\section{Contract}

For any aspiring GPwSI or PwSI it is important to be absolutely clear about the contractual obligations of the role. Some GPs or specialist nurses will be appointed by the PCO on a sessional basis whilst others will be employed as part of a service level agreement. Many contractual issues will be generic - such as pay scales and holiday entitlement - and these issues are dealt with in booklets provided by the British Medical Association ${ }^{8}$ or the Royal College of Nursing. ${ }^{9}$ Given that the PwSI may be in a fairly isolated role, it is important to clarify who will provide holiday and sickness cover.

In addition to general contractual issues ${ }^{8,9}$ the following questions should be considered:

- How is the position contracted? e.g. on a sessional basis as an employee, or as a service level agreement as an individual or as part of an agreement with an organisation.

- What is the length of the contract/tenure? This should be flexible, and should acknowledge that funding provision may change.

- What is the PwSI expected to provide as part of the contract? e.g. facilities, equipment, staff etc.

- What are the contracted hours and how are these divided between clinical and managerial tasks?

- Who is responsible for providing cover during unplanned absences? 


\section{Conclusions}

Practitioners with a Specialist Interest (PwSIs) in respiratory medicine working in the community have become increasingly common in the UK over the last few years. There is an increasing awareness by government authorities, and by healthcare professionals and their professional bodies, that PwSIs need to be accredited and regularly appraised in order to provide high quality services to patients. This Discussion document has outlined the important factors in the job description and terms of service which need to be addressed in order to ensure that PwSI services are of high quality, whilst at the same time providing maximal professional satisfaction for the PwSI. As we go to press, the DoH has now published Guidelines for the Accreditation of General Practitioners and Pharmacists with a Special Interest. ${ }^{10}$ These support the idea of accreditation by portfolio by a local panel of local stakeholders. Primary Care Trusts in England are directed to adhere to this guidance for new GPwSI's from 17.5.2007 and for existing GPwSI's by March 2009.

\section{Acknowledgements}

The authors wish to acknowledge the support of the GPIAG, and the financial support of AstraZeneca who funded the Working Party meeting. We gratefully acknowledge the members of the GPIAG Working Party, whose input into this paper was invaluable. The members of the Working Party were:

Dr Kevin Gruffydd-Jones (GPIAG)

Ms Monica Fletcher (Education for Health)

Dr Steve Holmes (GPIAG)

Mr Chris Town (Chief Executive, Greater Peterborough PCT)

Ms Jane Scullion (Royal College of Nursing)

Dr Richard Russell (British Thoracic Society)
Dr Noel O'Kelly (GPIAG)

Dr Dermot Ryan (GPIAG)

We also thank Tricia Bryant who produced the initial Working Party document.

\section{Conflict of interest declaration}

There were no conflicts of interest for the authors in the preparation of this paper.

\section{References}

1. Department of Health. Guidelines for the appointment of general practitioners with specialist interest in the delivery of clinical services - Respiratory medicine. DoH, London (2003)

2. Williams S, Ryan D, Price D, Langley C, Fletcher M, Everden P. General practitioners with a special clinical interest: a model for improving respiratory disease management. Br J Gen Pract 2002;52:838-43.

3. Gruffydd-Jones K. A proposal for the process of accreditation by portfolio for a GPwSI in respiratory medicine. Prim Care Resp J 2004;14(1):13-20. doi:10.1016/j.pcrj.2004.09.004

4. Holmes S, Gruffydd-Jones K. A proposal for the annual appraisal of and developmental support for GPwSIs in respiratory medicine. Prim Care Resp J 2005;14(3):161-5. doi 10.1016/j.pcrj.2005.03.001

5. http://www.gpiag.org/gpwsi/resourcepack. Accessed 16.2.07.

6. Ryan D, Levy M, Morris A, Sheikh A, Walker S. Management of allergic problems in primary care: time for a rethink? Prim Care Resp J 2005;14(4):195-203. doi:10.1016/j.pcrj.2005.01.003

7. http://www.educationforhealth.org.uk. Accessed 16/2/07

8. Advice leaflet for terms and conditions for salaried GPS for BMA members www.bma.org.uk/ap.nsf. Accessed 16/2/07.

9. RCN Guidance on Good Employment Practice published by the Royal College of Nursing. Available at www.ren.org.uk.

10. Implementing care closer to home: Convenient quality care for patients Part 3: The accreditation of GPs and Pharmacists with Special Interests. Department of Health, 2007. http://www.dh.gov.uk/en/Publicationsandstatistics/Publications/ PublicationsPolicyAndGuidance/DH_074430 (URL accessed 26.5.2007).

Available online at http://www.thepcrj.org 